\title{
Erratum to: Blood pressure response to catheter-based renal sympathetic denervation in severe resistant hypertension: data from the Greek Renal Denervation Registry
}

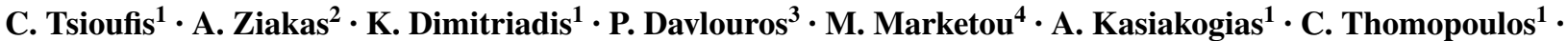 \\ D. Petroglou ${ }^{2}$ D. Tsiachris ${ }^{1} \cdot$ M. Doumas ${ }^{5}$ - E. Skalidis ${ }^{4}$ C. Karvounis ${ }^{2} \cdot$ D. Alexopoulos ${ }^{6} \cdot$ P. Vardas $^{4}$. \\ I. Kallikazaros ${ }^{7}$ C. Stefanadis ${ }^{1} \cdot$ V. Papademetriou ${ }^{1} \cdot$ D. Tousoulis ${ }^{1}$
}

Published online: 24 February 2017

(C) Springer-Verlag Berlin Heidelberg 2017

\section{Erratum to: Clin Res Cardiol DOI 10.1007/s00392-016-1056-z}

The original version of this article unfortunately contained a mistake.
The author name E. Skalidis was misspelled. The correct information is given in the authorline.

The original article has been corrected.

The online version of the original article can be found under doi:10.1007/s00392-016-1056-z.

\section{Tsioufis}

ktsioufis@hippocratio.gr

1 First Cardiology Clinic, Hippokration Hospital, University of Athens, 114 Vas.Sofias Ave., 11527 Athens, Greece

2 First Department of Cardiology, AHEPA University Hospital, Aristotle University of Thessaloniki, Thessaloniki, Greece

3 Department of Cardiology, Patras University Hospital, Patras, Greece

4 Cardiology Department, Heraklion University Hospital, Crete, Greece

5 2nd Propedeutic Department of Internal Medicine, Aristotle University, Thessaloniki, Greece

6 Department of Cardiology, Attikon University Hospital, Athens, Greece

7 Cardiology Department, Hippokration Hospital, Athens, Greece 